\title{
Inactivation of SERCA2 Cys674 accelerates aortic aneurysms by suppressing PPAR $\gamma$
}

\author{
Yumei Que ${ }^{1}$, Xi Shu ${ }^{1}$, Langtao Wang ${ }^{1}$, Sai Wang ${ }^{1}$, Pingping $\mathrm{Hu}^{1}$, and Xiaoyong Tong ${ }^{1}$ \\ ${ }^{1}$ Innovative Drug Research Center
}

August 28, 2020

\begin{abstract}
Background and Purpose Inactivation of Cys674 (C674) in the sarcoplasmic/endoplasmic reticulum Ca2+ ATPase 2 (SERCA2) by causing the accumulation of intracellular $\mathrm{Ca} 2+$ to activate calcineurin-mediated nuclear factor of activated T-lymphocytes (NFAT)/NF- $x$ B pathways, resulted in the phenotypic modulation of smooth muscle cells (SMCs) to accelerate angiotensin IIinduced aortic aneurysm. Our goal was to investigate the mechanism involved. Experimental Approach We used heterozygous SERCA2 C674S knock-in (SKI) mice, where half of C674 was substituted by serine, to represent partial irreversible oxidation of C674. The aortas of SKI mice and their littermate wild-type mice were collected for RNA sequencing, cell culture, protein expression, luciferase activity and aortic aneurysm analysis. KEY RESULTS Inactivation of C674 inhibited the promoter activity and protein expression of PPAR $\gamma$, which could be reversed by inhibitors of calcineurin or NF- $\varkappa$ B. Overexpression of PPAR $\gamma 2$ inhibited the phenotypic modulation of SKI SMCs. Pioglitazone, the activator of PPAR $\gamma$, blocked the activation of NFAT/NF- $\varkappa$ B, inhibited SMC phenotypic modulation, and ameliorated angiotensin II-induced aortic aneurysms in SKI mice. CONCLUSIONS AND IMPLICATIONS The inactivation of SERCA2 C674 promotes the development of aortic aneurysm by disrupting the balance between PPAR $\gamma$ and NFAT/NF- $\varkappa$ B. Our study highlights the importance of C674 redox status in regulating $\mathrm{PPAR} \Upsilon$ to maintain aortic homeostasis.
\end{abstract}

Title:Inactivation of SERCA2 Cys ${ }^{674}$ accelerates aortic aneurysms by suppressing PPAR $\gamma$

Running title: Inactivation of SERCA2 $\mathrm{Cys}^{674}$ suppresses PPAR $\curlyvee$

Authors

Yumei Que ${ }^{1}$, Xi Shu ${ }^{1}$, Langtao Wang ${ }^{1}$, Sai Wang ${ }^{1}$, Pingping $\mathrm{Hu}^{1}$, Xiaoyong Tong ${ }^{*}$

\section{Affiliations}

1. School of Pharmaceutical Sciences, Chongqing University, Chongqing, 401331, China

To whom correspondence should be addressed: Xiaoyong Tong, xiaoyongtong@cqu.edu.cn

Word count (excluding Methods, Abstract, References, Figure legends): 3937

Illustrations: 8 figures, 1 table.

\section{Abstract \\ Background and Purpose}

Inactivation of $\mathrm{Cys}^{674}$ (C674) in the sarcoplasmic/endoplasmic reticulum $\mathrm{Ca}^{2+}$ ATPase 2 (SERCA2) by causing the accumulation of intracellular $\mathrm{Ca}^{2+}$ to activate calcineurin-mediated nuclear factor of activated T-lymphocytes (NFAT)/NF- $x \mathrm{~B}$ pathways, resulted in the phenotypic modulation of smooth muscle cells 
(SMCs) to accelerate angiotensin II-induced aortic aneurysm. Our goal was to investigate the mechanism involved.

\section{Experimental Approach}

We used heterozygous SERCA2 C674S knock-in (SKI) mice, where half of C674 was substituted by serine, to represent partial irreversible oxidation of C674. The aortas of SKI mice and their littermate wild-type mice were collected for RNA sequencing, cell culture, protein expression, luciferase activity and aortic aneurysm analysis.

\section{KEY RESULTS}

Inactivation of $\mathrm{C} 674$ inhibited the promoter activity and protein expression of PPAR $\gamma$, which could be reversed by inhibitors of calcineurin or NF- $\varkappa \mathrm{B}$. Overexpression of PPAR $\gamma 2$ inhibited the phenotypic modulation of SKI SMCs. Pioglitazone, the activator of PPAR $\gamma$, blocked the activation of NFAT/NF- $x$ B, inhibited SMC phenotypic modulation, and ameliorated angiotensin II-induced aortic aneurysms in SKI mice.

\section{CONCLUSIONS AND IMPLICATIONS}

The inactivation of SERCA2 C674 promotes the development of aortic aneurysm by disrupting the balance between PPAR $\gamma$ and NFAT/NF- $\varkappa$ B. Our study highlights the importance of C674 redox status in regulating PPAR $\gamma$ to maintain aortic homeostasis.

\section{KEY WORDS}

SERCA2, aortic aneurysm, smooth muscle, phenotypic modulation, PPAR $\gamma, \mathrm{NFAT}, \mathrm{NF}-\varkappa \mathrm{B}$.

\section{Summary}

\section{'What is already known'}

The inactivation of SERCA2 $\mathrm{Cys}^{674}$ by causing the accumulation of intracellular $\mathrm{Ca}^{2+}$ to activate calcineurinmediated NFAT/NF- $x$ B pathways, results in SMC phenotypic modulation to accelerate aortic aneurysm.

\section{'What this study adds'}

- Inactivation of SERCA2 $\mathrm{Cys}^{674}$ causes the imbalance between PPAR $\gamma$ and NFAT/NF- $\chi \mathrm{B}$ in SMCs, leads to the sustained activation of NFAT/NF- $x \mathrm{~B}$.

- Activation of PPAR $r$ could restore this balance, thus suppresses SMC phenotypic modulation and aortic aneurysm formation caused by the inactivation of SERCA2 $\mathrm{Cys}^{674}$.

\section{'Clinical significance'}

- Increase levels of ROS irreversibly oxidize $\mathrm{Cys}^{674}$ in SERCA2 to promote the development of aortic aneurysm by suppressing PPAR $\gamma$.

- Activating PPARr contributes to controlling aortic aneurysm by restricting SMC phenotypic modulation.

- PPAR $Y$ and SERCA2 may be potential therapeutic targets for aortic aneurysm.

\section{Abbreviations}

C674, Cys ${ }^{674}$; Col I, collagen type I; Col III, collagen type III; CsA, cyclosporin A; DAPI, 4',6-diamidino2-phenylindole; FPKM, expected number of fragments per kilobase of transcript sequence per millions base pairs sequenced; HE, hematoxylin/eosin; ICAM1, intercellular adhesion molecule 1; LDLR ${ }^{-/-}$, LDL receptordeficient; MYOCD, myocardin; NFAT, nuclear factor of activated T-lymphocytes; OPN, osteopontin; PDTC, pyrrolidinedithiocarbamic acid; S674, Ser ${ }^{674}$; SERCA, sarco/endoplasmic reticulum calcium ATPase; SKI, SERCA2 C674S knock-in; SMCs, smooth muscle cells; VCAM1, vascular cell adhesion molecule 1; VVG, Verhoeff-Van Gieson; WT, wild-type

\section{1 | Introduction}


Aortic aneurysm is a fatal vascular disease, lack of effective drug treatment, surgery is the only choice. Most aortic aneurysms are asymptomatic before catastrophic complications such as dissection or rupture occur. Although surgery can stabilize large aneurysms, the recurrence rate and mortality rate are still high. Therefore, it is urgent to clarify the molecular mechanism of the occurrence and development of aortic aneurysm and determine the target of intervention.

Smooth muscle cells (SMCs) located in the media of aorta are key to the vascular remodeling of aortic aneurysm. Most SMCs are in the state of differentiation under physiological conditions to maintain the elasticity and tensile strength of the vascular wall. However, SMCs have significant phenotypic plasticity and can quickly adapt to environmental fluctuation signals. The transition of SMCs from a differentiated state to a dedifferentiated state is called phenotypic modulation, characterized by enhanced proliferation, migration, and expression of fibrotic and inflammatory proteins, which is the key cellular basis of aortic aneurysm (Ailawadi et al., 2009). Myocardin (MYOCD) is a differentiation marker of SMCs, and its deletion in SMCs causes severe aortic aneurysm (Tang et al., 2008). Matrix cytokine osteopontin (OPN) is a marker of dedifferentiation of SMCs, and its serum level predicts the progression of aortic aneurysm (Golledge et al., 2007). Collagen type I and type III ( Col I , Col III ) correlate well with the size of aortic aneurysm, and they are also SMC dedifferentiation markers (Meng et al., 2014; Wang et al., 2012a) .MMP2 degrades elastin and collagen (mainly Col IV in basal laminae), destroys vascular integrity, critical in angiotensin II-induced aortic aneurysm (Wang et al., 2012b). NF- $\varkappa$ B is a transcriptional factor and its activation favors its nuclear translocation to up-regulate the gene expression of MMP2, vascular cell adhesion molecule 1 (VCAM1) and intercellular adhesion molecule 1 (ICAM1), promotes SMC proliferation, migration, and inflammation (de Winther et al., 2005; Monaco et al., 2004; Tsai et al., 2017). NF- $x \mathrm{~B}$ is up-regulated in the aortic aneurysm wall, and inhibition of NF- $\chi \mathrm{B}$ decreases the size of aortic aneurysm (Miyake et al., 2007).

Sarco/endoplasmic reticulum calcium ATPase (SERCA) is a key enzyme to maintain calcium homeostasis by taking up $\mathrm{Ca}^{2+}$ from cytosol to sarcoplasmic reticulum and endoplasmic reticulum. SERCA2 is the main subtype of SERCA in the vasculature, and the $S$-glutathiolation of the amino acid residue Cys ${ }^{674}$ (C674) is key to increase the activity of SERCA2 under physiological conditions (Adachi et al., 2004; Tong et al., 2008), but this post-translational protein modification is prevented by the irreversible oxidation of C674 thiol in pathological situations characterised by high levels of ROS, such as aging, diabetes mellitus, atherosclerosis, and hypertension (Adachi et al., 2004; Liu et al., 2020; Qin et al., 2013; Ying et al., 2008), which are risk factors for aortic aneurysm. We have reported that the irreversible oxidation of C674 occurred broadly in mouse and human aortic aneurysms (Que et al., 2020). We used heterozygous SERCA2 C674S knock-in (SKI) mice, where half of C674 was substituted by serine, to represent partial irreversible oxidative inactivation of $\mathrm{C} 674$ under pathological conditions. The inactivation of $\mathrm{C} 674$ by causing the accumulation of intracellular $\mathrm{Ca}^{2+}$ to activate $\mathrm{Ca}^{2+}$-dependent calcineurin that promoted the nuclear translocation of nuclear factor of activated T-lymphocytes (NFAT) and NF- $x \mathrm{~B}$, therefore down-regulated the expression of MYOCD, while up-regulated the expression of OPN, MMP2, Col I, Col III, and VCAM1, accelerated SMC proliferation, migration and macrophage adhesion to SMCs, and exacerbated angiotensin II-induced aortic aneurysm (Que et al., 2020). We hypothesize that inactivation of SERCA2 C674 not only activates NFAT/NF- $x$ B, but also inhibits the protective factors that restrict the phenotypic modulation of SMCs, thus accelerating the occurrence of aortic aneurysm. This study was designed to elucidate the downstream targets of C674 in regulating SMC phenotypic modulation and aortic aneurysm.

\section{2 | Methods}

\section{1 | Animals}

All animal care and study protocols complied with the guidelines of the ethical use of animals and were approved by the Animal Care and Use Committee (Third Military Medical University, Chongqing, China). All efforts were made to minimize animal suffering and reduce the number of animals used. Animal studies are reported in compliance with the ARRIVE guidelines and with the recommendations made by the British Journal of Pharmacology (BJP) (Kilkenny et al., 2010; McGrath \& Lilley, 2015). Experimental protocols and design adhere to BJPguidelines (Curtis et al., 2015). 
Mice were bred and housed in the laboratory animal room at Chongqing University under specific pathogenfree conditions. Mice were kept in open polypropylene cages with clean chip bedding. The animal room was maintained at a controlled temperature $\left(22 \pm 3{ }^{\circ} \mathrm{C}\right)$ and a $12 \mathrm{hr}$ cycle of light and dark. Five mice in each cage were free to drink water and were fed on a regular diet $(0.3-0.8 \%$ sodium, $1.0-1.8 \%$ calcium, $0.6-1.2 \%$ phosphorus, Beijing Keao Xieli Feed Corporation, China) unless otherwise indicated. To obtain tissues, all mice were killed by i.p. injection with 2,2,2-tribromoethanol (250 mg $\mathrm{kg}^{-1}$, Sigma-Aldrich, Cat\# T48402).

\subsection{SERCA2 C674S knock-in mouse construct}

All mice used in this study were of C57BL/6J background (RRID: IMSR_JAX:000664 ) and were originally obtained from The Jackson Laboratory (Bar Harbor, Maine, USA). The construct of the SKI mouse (RRID: MGI:5780876 ) was generated as previously described (Thompson et al., 2014). Briefly, the genetic variation is TGT at $\mathrm{C} 674$ to TCC (Ser ${ }^{674}$ [S674]) in exon 14 of SERCA2. The presence of the C674S mutation was verified by sequencing of both genomic DNA and cDNA from heart. Homozygous SKI mice died before birth, so only heterozygous SKI mice that express 50\% of C674 and 50\% of S674 were used in this study, and their littermate wild-type (WT) mice without S674 were used as controls. For aneurysm studies, SKI mice were mated with LDL receptor-deficient (LDLR ${ }^{-/}$, The Jackson Laboratory, RRID: IMSR_JAX:002207) mice susceptible to aortic aneurysms to obtain SKI/LDLR ${ }^{+/-}$mice, which were further mated with $\mathrm{LDLR}^{-/}$ mice to obtain SKI/LDLR ${ }^{-/-}$mice. All mice used for aneurysm studies were derived from mating between $\mathrm{SKI} / \mathrm{LDLR}^{-/-}$mice and $\mathrm{LDLR}^{-/-}$mice, and the littermate $\mathrm{LDLR}^{-/-}$mice acted as controls.

\section{3 $\mid$ RNA sequencing and bioinformatics}

Aorta of 4-month-old male from LDLR ${ }^{-/-}$or SKI/LDLR/- mice were homogenized in Trizol for RNA isolation using the Direct-zol RNA MiniPrep Kit (Zymo Research, Irvine, CA). RNA sequencing was done in Novogene Bioinformatic Technology Co. Ltd (Beijing, China). Briefly, construction of RNA sequencing library was performed on an Illumina(r) Hiseq platform. Paired-end clean reads were aligned to the mouse genome (GRCm38, RRID:SCR_002344), using TopHat v2.0.12. The reads numbers mapped to each gene were quantified using HTSeq v0.6.1. The expected number of fragments per kilobase of transcript sequence per millions base pairs sequenced (FPKM) of each gene was calculated based on the length of the gene and reads count mapped to this gene that reflects the relative gene expression level. Differentially expressed genes between $\mathrm{LDLR}^{-/}$and SKI/LDLR ${ }^{-/}$mice were analysed using DESeq R package (1.18.0). Gene set enrichment analysis was performed on differentially expressed genes (altered $>1.5$ fold) to test for enrichment of specific ontologies (Subramanian et al., 2005), and the Kyoto encyclopedia of genes and genomes (http://www.genome.jp/kegg/) was used to perform pathway analysis.

\section{4 | Real-time quantitative PCR}

Total RNA was isolated from aorta using TRIzol reagent (Invitrogen, Cat\# 15596026) and retro-transcribed to cDNA using cDNA PCR kit (Takara Bio Inc., Cat\# RR037Q). Real-time quantitative PCR was performed using SYBR-Green-based detection (Takara Bio Inc., Cat\# RR420L) with primers commercially synthesized (Sangon Biotech, China). The primer sequences from 5' to 3' are: 1) PPAR $\gamma 2$ forward, TCGCTGATGCACTGCCTATG, PPAR $\gamma 2$ reverse, GAGAGGTCCACAGAGCTGATT; 2) internal control $\beta$-actin forward, AGAGGGAAATCGTGCGTGAC, $\beta$-actin reverse, CAATAGTGATGACCTGGCCGT. Real time quantitative $\mathrm{PCR}$ was performed using the following cycling conditions: denaturation, annealing, and extension at $95^{\circ} \mathrm{C}, 57^{\circ} \mathrm{C}$ and $72^{\circ} \mathrm{C}$ for $10 \mathrm{~s}, 30 \mathrm{~s}$, and $10 \mathrm{~s}$, respectively, for 40 cycles. Relative expression of PPAR $\gamma 2$ was analysed using the comparative $\mathrm{Ct}$ method $\left(2^{-\mathrm{Ct}}\right)$.

\section{5 | Aortic SMC culture}

Aortic SMCs were isolated from 8-week-old male WT and SKI mice as previously described (Tong et al., 2015) and cultured in DMEM supplemented with 10\% FBS (ExCell Bio, Cat\# FSP500), 100 U/mL penicillin and $100 \mu \mathrm{g} / \mathrm{mL}$ streptomycin at $37^{\circ} \mathrm{C}$ in a humidified atmosphere containing $5 \% \mathrm{CO}_{2}$. SMC phenotype was confirmed by $\alpha$-smooth muscle actin immunostaining. Cells from passages 3 to 8 were used. All SMCs used were isolated from C57BL/6J background due to the SMCs from $\mathrm{LDLR}^{-/}$background were infeasible for 
further studies after subculture. Using different genetic background for mechanism study is commonly used in literature (Nakao et al., 2017). In some experiments, SMCs were administrated with pioglitazone $(10 \mu \mathrm{M}$, MCE, Cat\# U72107), or pyrrolidinedithiocarbamic acid (PDTC, ammonium salt, $10 \mu \mathrm{M}$, Macklin, Cat\# A800469) for $48 \mathrm{hr}$, before being collected for Western blot analysis or cell function studies. DMSO served as a solvent control, and its final concentration was less than $0.1 \%$.

\section{6 | Western blot}

Aortas or SMCs cultured in 0.2\% FBS DMEM overnight were lysed in RIPA buffer (Enogene, Cat\# E1WP106). Experimental details of Western blots are in accordance with BJP guidelines (Alexander et al., 2018). In some experiments, protein was extracted from the nucleus and cytoplasm by using the protein extraction kit (Beyotime Biotechnology, Cat\# P0027). Proteins were separated by SDS-PAGE electrophoresis using standard methods, transferred to PVDF membrane, and immunoblotted with specific antibodies overnight at $4{ }^{\circ} \mathrm{C}$ against PPAR $\curlyvee$ (detect both PPAR $\gamma 1$ and PPAR $\gamma 2$, Engene, Cat\# E2A6073, $58 \mathrm{kDa}$, RRID:AB_2861246 ), MYOCD (Abcam, Cat\# Ab107301,100 kDa, RRID:AB_11128102 ), OPN (Proteintech, Cat\# 22952-1-AP, $44 \mathrm{kDa}$, RRID:AB_2783651 ), MMP2 (Proteintech, Cat\# 10373-2-AP, $64 \mathrm{kDa}$, RRID:AB_2250823), Col I (Engene, Cat\# E110154C, $139 \mathrm{kDa}, R R I D: A B_{-2} 2861247$ ), Col III (Abcam, Cat\# Ab7778, $139 \mathrm{kDa}$, RRID:AB_306066 ), p65NF-xB (Engene, Cat\# E011014, 65 kDa, RRID:AB_2861239), phosphorylated p65NF- $x \mathrm{~B}$ (Engene, Cat\# E1A2006V, $65 \mathrm{kDa}, R R I D: A B_{-2861248}$ ), VCAM1 (Engene, Cat\# E90279, $72 \mathrm{kDa}$, RRID:AB_2861237), ICAM1 (Engene, Cat\# E301187, $82 \mathrm{kDa}$, RRID:AB_2861238 ), NFAT4 (Abcam, Cat\# Ab93628, $117 \mathrm{kDa}$, RRID:AB_10714571 ), GAPDH (Engene, Cat\# E1C604, 37 $\mathrm{kDa}$, RRID: AB_2814765 ), Histone H3 (Engene, Cat\# E11-0434B, $17 \mathrm{kDa}, A B_{-} 2861240$ ), followed by incubation with HRP-conjugated goat anti-rabbit secondary antibody (Sino Biological Inc., Cat\# SSA003, RRID:AB_2814815) $1 \mathrm{hr}$ at room temperature. Proteins were visualized with an X-ray film system (Fujifilm, Japan) or ChemiDoc Touch System (Bio-Rad, USA). Band density was quantified by NIH ImageJ software (RRID:SCR_003070, https://imagej.net/) and normalized to GAPDH or Histone H3.

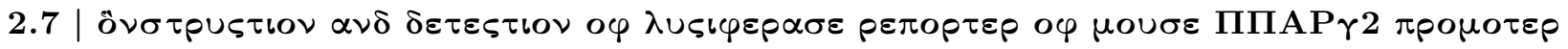

There are two NFAT binding (TTTTCC) sites in the human PPAR $\gamma 2$ promoter (distal -299 to -294 bp and proximal -146 to $-141 \mathrm{bp}$ ) to fully regulate its expression in adipocytes (Kim et al., 2010) and hepatoma cells (Yang et al., 2003). However, these two NFAT binding sites haven't been reported in mouse PPAR ${ }^{2}$ promoter. We compared the sequence of mouse and human PPAR $\gamma 2$ promoters from -2000 to $+45 \mathrm{bp}$ and found that there were two potential NFAT binding sites in mouse PPAR 22 promoter, of which -395 to -390 bp (TTTTCT) was homologous to human distal NFAT site, and -238 to -233 bp (TTTTCC) was homologous to human proximal NFAT site. These two NFAT binding sites also covered the NF- $x \mathrm{~B}$ binding sequence (TTTCC). Mouse liver genomic DNA was extracted as template to amplify the promoter sequence of PPAR $\gamma 2$ from -630 to $+45 \mathrm{bp}$ that contains the putative NFAT/NF- $x \mathrm{~B}$ binding sites and its 5'- untranslated region (GenBank, AY243584.1) with forward primer (5'AGAACATTTCTCTATCGATA GGTACC TAGAATTTGGATAGCAGTAAC3') containing Kpn I digestion site and reverse primer containing Hind III digestion site (5'ACCAACAGTACCGGAATGCCAAGCTT AACAGCATAAAACAGAGATTTG3').

The amplified DNA fragments were digested by Kpn I (BioLabs, Cat\# R0142S) and Hind III (BioLabs, Cat\# R0104S), and purified. The pLG4.19 basic plasmid vector (Promega, Cat\# E6741) containing firefly luciferase sequence was digested with Kpn I and Hind III to obtain the linearized vector. A PPAR 2 -pLG4.19 plasmid containing mouse PPAR 2 promoter $(-630$ to $+45 \mathrm{bp})$ and firefly luciferase was obtained by mixing purified PPAR $\gamma 2$ promoter fragment, linearized pLG4.19 and assembly reagent (TransGene Biotech, Cat\# CU101) for $15 \mathrm{~min}$ at $50{ }^{\circ} \mathrm{C}$. PCR and sequencing confirmed that the PPAR $\gamma 2-$ pLG4.19 firefly luciferase plasmid was constructed correctly. Large scale amplification of PPAR 2 2-pLG4.19 plasmid was carried out in TransT1 Escherichia coli. For mouse PPAR $\gamma 2$ promoter activity assay, $1 \mu \mathrm{g}$ PPAR $\gamma 2$-pLG4.19 firefly luciferase and $100 \mathrm{ng}$ pRL-TK renilla luciferase plasmid control (Promega, Cat\# E2241) were cotransfected into SMCs $\left(8 \times 10^{5}\right.$ cells per $60 \mathrm{~mm}$ dish) using SuperFect Transfection Reagent (Qiagen, Germany, Cat\# 301305) as per manufacturer's recommendations. Luciferase activities were measured using the Dual-Luciferase reporter assay kit (Beyotime Biotechnology, Cat\# RG027) $72 \mathrm{hr}$ after transfection. The 
ratio of firefly to renilla luciferase luminescence was calculated as indicative of PPAR $\gamma 2$ promoter activity. In some experiments, SMCs were treated cyclosporin A (CsA, $1 \mu \mathrm{M})$ or PDTC $(10 \mu \mathrm{M})$ for $24 \mathrm{hr}$ before measuring the activity of PPAR $\gamma 2$ promoter.

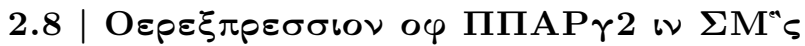

The PPAR 2 plasmids (ORIGENE, Cat\# MR208132) or its control CMV-GFP plasmids (ORIGENE, Cat\# PS100040) were transfected into SKI SMCs according to Effectene@ Transfection Reagent Handbook (Qiagen, Cat\#301305). The fluorescence intensity of GFP was observed by fluorescence microscope (Leica, DMi8) to evaluate the transfection efficiency. SMCs were collected $48 \mathrm{hr}$ later for protein analysis and cell function studies.

\section{9 | Cell proliferation assay}

A tetrazolium-based non-radioactive proliferation assay kit (Quick Cell Proliferation Assay Kit II, BioVision, Cat\# K301-500) was used to determine the cell number according to the manufacturer's protocol (Tong et al., 2016). SKI SMCs were seeded in 96-well plate at a density of $5 \times 10^{3}$ cells per well in DMEM supplemented with $0.2 \%$ FBS overnight. Cell proliferation was stimulated by medium supplemented with $10 \%$ FBS, and $0.2 \%$ FBS medium was used as a control. PDTC $(10 \mu \mathrm{M})$ or pioglitazone $(10 \mu \mathrm{M})$ was added to the culture media. DMSO served as a solvent control. Culture medium with high or low serum was changed daily. The cell number in each well was determined $72 \mathrm{hr}$ later.

\subsection{0 | Wounded monolayer migration assay}

SKI SMCs were pretreated with PDTC $(10 \mu \mathrm{M})$ or pioglitazone $(10 \mu \mathrm{M})$ for $48 \mathrm{hr}$ before migration assay (Tong et al., 2016). DMSO acted as solvent control. $10^{6}$ cells/well in 12 -well plates were seeded in $0.2 \%$ FBS DMEM overnight to reach confluence, then scratch wounds were applied to SMC monolayer with a pipette tip. Immediately after scratching, the cells were treated with $10 \%$ FBS DMEM to stimulate cell migration. Photographs were taken at $0 \mathrm{hr}$ and $6 \mathrm{hr}$ at three fixed locations along the scratch with a light microscope and analyzed using NIH Image J software.

\subsection{1 | Macrophage adhesion assay}

Bone marrow-derived mononuclear cells were isolated from the femurs of WT mice and cultured in highglucose DMEM supplemented with 10\% FBS and 20\% L929-conditioned medium for 5 days; at this point, adherent mononuclear cells are macrophages. SKI SMCs were pretreated with PDTC $(10 \mu \mathrm{M})$ or pioglitazone $(10 \mu \mathrm{M})$ for $48 \mathrm{hr}$ before macrophage adhesion assay. DMSO acted as solvent control. For macrophage adhesion assay (Tong et al., 2016), SMCs were plated in 12-well plate, at a density of $10^{6}$ cells/well, in $0.2 \%$ FBS DMEM until confluent. Macrophages $\left(5 \times 10^{5}\right.$ cells/well) were added to SMCs and incubated for $1 \mathrm{hr}$; the media was removed and SMCs washed 3 times with PBS to remove unbound macrophages. Four images were taken in each well and the number of bound macrophages was counted per image area.

\subsection{Angiotensin II-induced aortic aneurysm}

Male WT and SKI mice in LDLR ${ }^{-/-}$background at 7 weeks old (18-22 g) were fed on a Western diet (21\% w/w milk fat, $0.15 \%$ w/w cholesterol, Beijing Keao Xieli Feed Corporation) for 7 days and then received subcutaneous implantation of ALZET osmotic pumps (Model 2004, DURECT Corporation, USA), delivering angiotensin II (1.44 mg* $\mathrm{kg}^{-1} *$ day $^{-1}$, dissolved in saline)(Bhamidipati et al., 2014). Before implantation, mice were given buprenorphine $\left(0.08 \mathrm{mg}^{*} \mathrm{~kg}^{-1}\right.$, s.c.) as analgesic, and during pump implantation, they were anaesthetised with isoflurane (1.5\%) inhalation. The mouse back was depilated with depilatory ointment. After disinfection with iodophor, the pump was implanted subcutaneously into the back of the mouse. The incision was sealed with sterile sutures and disinfected with iodophor. Buprenorphine $\left(0.08 \mathrm{mg} \mathrm{kg}^{-1}\right.$, s.c., every $8 \mathrm{hr}$ ) was given to the mouse the first day after surgery. The mice were monitored every day in the first week after operation. Seven days after angiotensin II infusion, SKI mice were randomly given pioglitazone $\left(15 \mathrm{mg}^{*} \mathrm{~kg}^{-1 *}\right.$ day $^{-1}$, suspended in $200 \mu \mathrm{L}$ sterile water, $\left.n=11\right)$ or solvent control sterile water $(200 \mu \mathrm{L}, n$ $=11$ ), and WT mice were given sterile water $(200 \mu \mathrm{L}, n=11)$. They were given by gavage once a day. 
Western diet was applied during the whole experiment. 28 days after the infusion of angiotensin II, mice were killed and perfused with ice-cold PBS, and then the connective tissue was removed to expose the whole aorta and its collateral branches and digital images were acquired for aneurysm analysis using an Olympus microscope (model SZX16). Aneurysms are defined as enlargements $>50 \%$ diameter compared to littermate controls without angiotensin II infusion. The composition of aneurysms was evaluated according to the type of aneurysm, including the ruptured aneurysm, unruptured aneurysm, and non-aneurysm. The survival rate and incidence of aneurysm were monitored and calculated. The number of aneurysms per mouse was counted. The length of aneurysm was measured according to the scale with CellSens standard software 1.12 (RRID:SCR_014551, Olympus).

\subsection{3 | Histology and immunohistochemistry}

Aneurysms were carefully dissected and fixed in $4 \%$ paraformaldehyde overnight, followed by $30 \%$ sucrose solution for $24 \mathrm{hr}$ before embedding in optimum cutting temperature compound to prepare serial frozen sections at a thickness of $7 \mu \mathrm{m}$. Remaining portions of the aorta were snap-frozen in liquid nitrogen and stored at $-80{ }^{\circ} \mathrm{C}$ for protein analysis. Twenty serial sections of the largest aneurysm for each mouse were prepared and stained individually with hematoxylin/eosin (HE), Verhoeff-Van Gieson (VVG) and Masson's trichrome for morphological analysis, elastin assessment and collagen deposition, respectively. The area of aneurysm was measured according to the scale with CellSens standard software. The collagen deposition was scored on a scale of 1 to 4 , with $1-<25 \%$ area in medium layer was occupied by collagen, 2-25-50\% collagen deposition, 3-50-75\% collagen deposition, and 4- > 75\% collagen deposition. Elastin lamellae were evaluated using a grade of I to IV, with I- intact internal elastic lamina, II- mild elastin fragmentation, III- severe elastin digestion, and IV- severe elastin digestion with visible ruptured sites. The collagen deposition and elastin degradation scores among different observers blinded to the experimental groups were comparable; data are expressed as the mean value of different observers' scores.

\subsection{Measurement of plasma cholesterol and triglycerides}

Mice were sacrificed and blood was drawn from the right atrium into EDTA-containing tubes, for lipid measurements. Plasma was prepared via centrifugation at $850 \times \mathrm{g}$ for $15 \mathrm{~min}$ at $4{ }^{\circ} \mathrm{C}$ and stored at $-20{ }^{\circ} \mathrm{C}$. Measurements were carried out using the Infinity Cholesterol and Triglycerides measurement kits (Thermo Scientific, USA, Cat\# TR13421, Cat\# TR22421), based on the absorbance of samples normalized to the absorbance of a known concentration of a calibrator provided with the kit.

\subsection{5 | Data and statistical analysis}

The data and statistical analysis comply with the recommendations of the $B J P$ on experimental design and analysis in pharmacology (Curtis et al., 2018). All studies were designed to generate groups of equal size, using randomization and blinded analysis. Group size is the number of independent values, and statistical analysis was carried out using these independent values. Sample sizes in each group subjected to statistical analysis were determined based on our previous studies, preliminary results, and power analysis (Curtis et al., 2015). Statistical analysis was undertaken only using these independent values with $n$ [?] 5. Fold change over control was used in Western blot and gene analysis to avoid the larger variation among different experiments. The mean values of the control group were normalized to 1 . In the figures, the $\mathrm{Y}$ axis shows the ratio of the experimental group to that of the corresponding matched control values and is labelled as "fold matched control values." All results were expressed as mean +- SEM. Statistical analysis was performed using GraphPad Prism 7.00 (RRID:SCR_002798, http://www.graphpad.com/). Unpaired Student's t test was used to analyse data from two groups. Differences among multiple groups were analysed by one-way ANOVA with Bonferroni post hoc analysis. Chi-square test was used to analyse the composition of aneurysm, the elastin degradation, and the incidence of aneurysm. Survival rate was noted in Kaplan-Meier analysis and comparisons were performed using the log-rank test. For angiotensin II-induced aortic aneurysm, data analysis (survival rate, incidence, elastin degradation, number of aneurysms) was performed on 11 mice in each group. In the characteristic analysis of aneurysms (diameter, area, length, collagen deposition), mice died of ruptured aneurysms (4 SKI mice treated with solvent control) and mice without aneurysms (3 WT 
mice treated with solvent control and 2 SKI mice treated with pioglitazone) were excluded. For determining whether groups differ, the level of probability was set at $P<.05$ to constitute the threshold for statistical significance.

\subsection{6 | Materials}

Angiotensin II was supplied by BACHEM (Cat\# H-1705.0100).Pioglitazone was supplied by MCE (Cat\# U72107). PDTC was supplied by Macklin (Cat\# A800469). Buprenorphine was supplied by Sigma-Aldrich (Cat\# BP1062). CsA was supplied by MCE (Cat\# HY-B0579).

\subsection{7 | Nomenclature of targets and ligands}

Key protein targets and ligands in this article are hyperlinked to corresponding entries in http://www.guidetopharmacology.org, the common portal for data from the IUPHAR/BPS Guide to PHARMACOLOGY (RRID:SCR_013077) (Harding et al., 2018), and are permanently archived in the Concise Guide to PHARMACOLOGY 2019/20 (Alexander et al., 2019).

\section{3 | Results}

\section{$3.1 \mid$ I

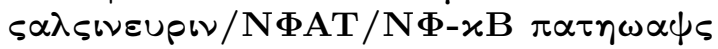

Our previous study indicates that the inactivation of $\mathrm{C} 674$ by causing the accumulation of intracellular $\mathrm{Ca}^{2+}$ to activate calcineurin-mediated NFAT/NF- $x \mathrm{~B}$ pathways, resulted in SMC phenotypic modulation to accelerate aortic aneurysm. In order to study the mechanism, we first performed RNA sequencing. Bioinformatics analysis showed that inactivation of $\mathrm{C} 674$ had a wide range of effects on gene expression, and a variety of signaling pathways were affected, among which PPAR signaling pathway was the most closely related to the SMC phenotypic modulation (Figure 1A). PPAR belongs to the nuclear receptor superfamily, including PPAR $\alpha$, PPAR $\beta$ and PPAR $\gamma$. FPKM values showed that inactivation of 6744 had no effect on $\Pi \Pi A P a$ (WT: $1.49 \pm 0.28$; SKI: $1.06 \pm 0.11$ ) and ППАР $\beta$ (WT: $25.13 \pm 2.22$; SKI: $29.74 \pm 2.93$ ), but decreased PPAR $\gamma 2$ (WT: $5.65 \pm 0.68$; SKI: $2.44 \pm 0.21$ ). The down-regulation of PPAR $\gamma 2$ was further confirmed at both mRNA level by real-time quantitative PCR (Figure 1B) and protein level by Western blot (Figure 1C) in aorta of SKI mice.

Activation of calcineurin or NF- $x \mathrm{~B}$ inhibited the expression of PPAR $\gamma$ in adipocytes (Liu et al., 2007) and pulmonary artery SMCs (Xie et al., 2017). To explore whether C674 inactivation regulates the transcription of PPAR $\gamma 2$ by affecting its promoter activity, we constructed a mouse PPAR $\gamma 2$ promoter from -630 to $+45 \mathrm{bp}$, containing NFAT/NF- $x$ B binding sites. Compared with WT SMCs, the activity of PPAR $\gamma 2$ promoter in SKI SMCs was down-regulated (Figure 1D), which could be reversed by either calcineurin inhibitor CsA or NF- $\varkappa \mathrm{B}$ inhibitor PDTC (Figure 1E). Correspondingly, compared with WT SMCs, the protein expression of PPAR $\gamma$ was down-regulated in SKI SMCs, which could be reversed by either CsA (Figure 1F) or PDTC (Figure 2A), suggesting that PPAR $\gamma$ might be the downstream target of calcineurin/NFAT/NF- $\varkappa \mathrm{B}$ pathways. We have reported that inactivation of 674 decreased the expression of MYOCD, increased the expression of OPN, MMP2, Col I, Col III, phosphorylated p65NF- $x$ B, VCAM1, and ICAM1, accelerated SMC proliferation, migration and macrophage adhesion to SMCs, and all these characters could be reversed by CsA (Que et al., 2020). CsA inhibits the nuclear translocation of NFAT4 and NF- $x B$ in SKI SMCs (Que et al., 2020). Here we showed that NF- $\chi \mathrm{B}$ inhibitor PDTC had similar effect to CsA in SKI SMCs (Figure 2), further supporting that the activation of $\mathrm{NF}-\varkappa \mathrm{B}$ pathway caused by inactivation of $\mathrm{C} 674$ promoted $\mathrm{SMC}$ phenotypic modulation. These results indicate that inactivation of 6674 promotes the binding of NFAT/NF- $x \mathrm{~B}$ to PPAR $\gamma 2$ promoter by increasing the nuclear translocation of NFAT/NF- $x \mathrm{~B}$, thus negatively regulating the transcription of PPAR $\gamma 2$, resulting in the decrease of PPAR $\gamma$ protein expression.

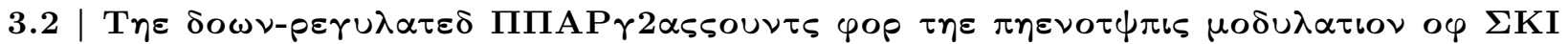 $\Sigma M^{\prime \prime} s$}

$\Pi \Pi A P \gamma$ prevents the dedifferentiation of SMCs, restricts SMC proliferation, migration and the activation 
of inflammatory pathway (Abe et al., 2003; Hamblin et al., 2009). The two isoforms of PPAR $\gamma$, PPAR 1 and PPAR $\gamma 2$, differ at their $\mathrm{N}$ terminal. The protective effect of PPAR $\gamma 1$ on the phenotypic modulation of SMCs was studied (Halabi et al., 2008; Hu et al., 2008; Meredith et al., 2009). However, the role of PPAR $2^{2}$ in the regulation of SMC phenotype is still unclear. Although PPAR $\gamma 2$ has 30 more amino acids than PPAR $\gamma 1$, it has similar DNA binding area and ligand binding region to PPAR $\gamma 1$ (Werman et al., 1997). In SKI SMCs, overexpression of PPAR $\gamma 2$ increased the expression of MYOCD and decreased the expression of OPN, MMP2, Col I, Col III, phosphorylated p65NF- $x$ B, VCAM1 and ICAM1 (Figure 3A), and inhibited cell proliferation, migration, and macrophage adhesion to SMCs (Figure 3B-D). This provides direct evidence that PPAR 22 inhibits the phenotypic modulation of SMCs, and indicates that the decrease of PPAR 2 is accountable for the phenotypic modulation of SKI SMCs.

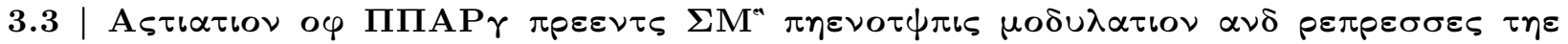

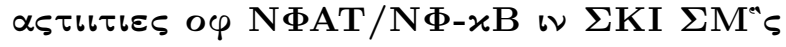

Next, we used PPAR $\gamma$ activator pioglitazone to compensate for the down-regulation of PPAR $\gamma$ expression in SKI SMCs. Activation of PPAR $\gamma$ by pioglitazone in SKI SMCs, similar to the overexpression of PPAR $\gamma 2$, also increased the expression of MYOCD and decreased the expression of OPN, MMP2, Col I, Col III, phosphorylated p65NF- $x \mathrm{~B}$, VCAM1 and ICAM1 (Figure 4A), and inhibited cell proliferation, migration, and macrophage adhesion to SMCs (Figure 4B-D), which further confirmed the contribution of the downregulated PPAR $\gamma$ in promoting the phenotypic modulation of SKI SMCs.

Though PPAR $\gamma$ could be a downstream target of calcineurin/NFAT/NF- $\chi \mathrm{B}$ signaling pathways, in return it could also interfere with NFAT and NF- $\varkappa$ B to repress their activities (Blanquart et al., 2003). Inactivation of C674 increases the nuclear translocation of NFAT4 and NF- $x \mathrm{~B}$ to be active (Que et al., 2020). As shown in Figure 5, the activation of PPAR $\gamma$ by pioglitazone could inhibit the nuclear translocation of NFAT4 and NF- $x$ B in SKI SMCs, suggesting the mutual negative regulation between NFAT/NF- $x \mathrm{~B}$ and PPAR $\gamma$ in SMCs.

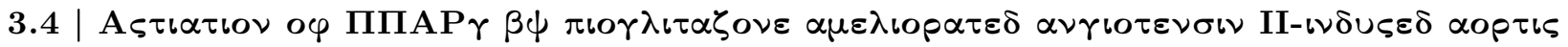 $\alpha \nu \varepsilon \cup \rho \psi \sigma \mu$ iv $\Sigma K I \mu l s \varepsilon$}

Activation of PPAR $\gamma$ by pioglitazone protected the formation and rupture of experimental aortic aneurysms in mice (Golledge et al., 2010; Shimada et al., 2015). Next, we used LDLR ${ }^{-/-}$background mice for angiotensin II-induced aortic aneurysm analysis, and pioglitazone or solvent control administered 7 days after angiotensin II infusion (Figure 6A). After 28 days of angiotensin II infusion, there was no difference in body weight, plasma levels of triglyceride and cholesterol among WT mice treated with solvent control, SKI mice treated with solvent control, and SKI mice treated with pioglitazone (Table 1).

During 28-day angiotensin II infusion, all WT mice treated with solvent control (11 out of 11) and SKI mice treated with pioglitazone (11 out of 11) survived, while 7 out of 11 SKI mice treated with solvent control survived (Figure 6B). As shown in Figure 6C, 8 out of 11 WT mice treated with solvent control developed aortic aneurysms. In contrast, all SKI mice treated with solvent control (11 out of 11) developed aortic aneurysms, in which 4 of them had early death due to the aneurysm rupture. Pioglitazone slightly decreased the incidence of aortic aneurysms in SKI mice (9 out of 11) comparable to WT mice treated with solvent control. Compared with WT mice treated with solvent control, SKI mice treated with solvent control had severer aortic aneurysm, of which 4 out of 11 had ruptured aneurysms. Pioglitazone ameliorated aortic aneurysms in SKI mice with improved composition of aortic aneurysm comparable to WT mice treated with solvent control (Figure 6D). Accordingly, SKI mice treated with solvent control had a higher grade of elastin degradation and a slight increase of collagen deposition than WT mice treated with solvent control, and pioglitazone treatment could reverse the increased elastin degradation and collagen deposition in SKI mice comparable to WT mice treated with solvent control (Figure 6E\&F).

Mice (4 out of 11 SKI mice treated with solvent control) that died of aneurysm rupture and mice without aneurysm (3 out of 11 WT mice treated with solvent control and 2 out of 11 SKI mice treated with pioglitazone) during the 28-day angiotensin II infusion were excluded from aneurysm size analysis. The largest 
aneurysm in each mouse was analyzed. Compared with WT mice treated with solvent control, in SKI mice treated with solvent control, the diameter of aneurysm, the outer area of aneurysm, and the media area with aneurysm, all showed a trend of increase, while the lumen area showed a trend of decrease, and all these characters of SKI mice could be reversed by pioglitazone treatment (Figure 7A). The length and number of aneurysms were significantly larger in SKI mice treated with solvent control compared with WT mice treated with solvent control, while pioglitazone treatment reduced the length and number of aneurysms in SKI mice (Figure 7B\&C).

In SKI non-aneurysmal aortas infused with angiotensin II, though pioglitazone had no effect on the protein expression of PPAR $\gamma$ compared with solvent control, it did increase the expression of MYOCD and decrease the expression of OPN, MMP2, Col I, Col III, phosphorylated p65NF- $\varkappa \mathrm{B}$, VCAM1, and ICAM1 (Figure 8), further supporting the regulation of PPAR $\gamma$ on these aneurysm-related proteins in vivo . These results indicated that inactivation of $\mathrm{C} 674$ by downregulation of PPAR $\gamma$ to promote the phenotypic modulation of SMCs, thus aggravated angiotensin II-induced aortic aneurysms, and pioglitazone by suppressing these aneurysm-related proteins to improve aortic aneurysms.

\section{4 | Discussion and conclusion}

SERCA2 is the main subtype of SERCA in the vascular system to maintain calcium homeostasis. The C674 in the SERCA2 is evolutionarily conserved. We previously reported that the irreversible oxidation of C674 occurred broadly in mouse and human aortic aneurysms (Que et al., 2020). Using SKI mice to mimic the persistent oxidative inactivation of $\mathrm{C} 674$, we find that inactivation of $\mathrm{C} 674$ promotes the development of aortic aneurysm by inducing SMC phenotypic modulation, which is caused by the accumulation of intracellular $\mathrm{Ca}^{2+}$ that activates $\mathrm{Ca}^{2+}$-dependent calcineurin to promote the nuclear translocation of NFAT and NF- $x \mathrm{~B}$ to be active. In this study, we found that the enhanced activation of NFAT/NF- $\varkappa$ B pathways by C674 inactivation suppressed the expression of PPAR $\gamma$. In SKI mice, activation of PPAR $\gamma$ blocked the activation of NFAT/NF- $\varkappa$ B, inhibited SMC phenotypic modulation, and ameliorated angiotensin II-induced aortic aneurysms, suggesting that the down-regulation of PPAR $\curlyvee$ by C674 inactivation is critical to SMC phenotypic modulation and is responsible for the accelerated aortic aneurysm in SKI mice. Our result indicates that the redox state of $\mathrm{C} 674$ in the SERCA2 is pivotal in maintaining the balance between calcineurin-mediated $\mathrm{NFAT} / \mathrm{NF}-x \mathrm{~B}$ pathways and PPAR $\gamma$. Inactivation of $\mathrm{C} 674$ promotes the development of aortic aneurysm by inducing SMC phenotypic modulation, which is caused by the imbalance between the enhanced activation of calcineurin-mediated NFAT/NF- $x$ B pathways and the reduced expression of PPAR $\gamma$.

PPAR $\gamma$ is a key downstream target of C674 inactivation, which triggers the phenotypic modulation of SMCs and induces aortic aneurysm. PPAR $\gamma$ has two major isoforms, PPAR $\gamma 1$ and PPAR $\gamma 2$, whose differ at $\mathrm{N}$ terminal. The expression levels of PPAR $\gamma 1$ and PPAR $\gamma 2$ are similar in human SMCs (Limor et al., 2008). PPAR $\gamma$ prevents the dedifferentiation of SMCs, restricts SMC proliferation, migration and the activation of inflammatory pathways (Hamblin et al., 2009). In vascular SMCs, dysregulation of PPAR $\gamma$ upregulates protein expression of inflammatory factors, MMPs and OPN, enhances cell proliferation and migration (Halabi et al., 2008; Hasan et al., 2015; Meredith et al., 2009). Overexpression of PPAR $\gamma 1$ attenuates atherosclerosis and stabilizes vulnerable plaques (Hu et al., 2008). Lack of SMC PPAR $\gamma 1$ enhances SMC proliferation and migration, promotes the vascular remodeling (Halabi et al., 2008; Meredith et al., 2009), confirming the protective effect of PPAR $\gamma 1$ in SMC phenotypic modulation. Rosiglitazone and pioglitazone, activators of both PPAR $\gamma 1$ and PPAR $\gamma 2$, are beneficial in experimentally reducing aortic aneurysms (Blanquart et al., 2003; Chen et al., 2015). The polymorphism of PPAR ${ }^{2}$ (Pro ${ }^{12 \mathrm{Ala}}$ ) is associated with a higher risk of cardiovascular diseases, particularly myocardial infarction (Li et al., 2015). However, it's not clear whether PPAR $\gamma 2$ contributes to SMC phenotypic modulation and aortic aneurysm formation. The RNA sequencing result indicated that PPAR 22 was down-regulated by C674 inactivation. In SKI SMCs, overexpression of PPAR $\gamma 2$ inhibited the expression of aneurysm-related proteins and restricted SMC phenotypic modulation. Our data provides a direct evidence of PPAR $\gamma 2$, similar to PPAR $\gamma 1$, in restricting SMC phenotypic modulation. The reduced PPAR $\gamma 2$ is responsible for SKI SMC phenotypic modulation. This highlights the critical role of PPAR $\curlyvee$ regulated by the redox state of $\mathrm{C} 674$ in maintaining aortic homeostasis. 
NFAT and NF- $\varkappa$ B share a number of properties, including similar DNA binding domains and rapid nuclear translocation to response external stimulus (Serfling et al., 2004). Activation of calcineurin or NF- $x \mathrm{~B}$ inhibits the expression of PPAR $\gamma$ in adipocytes (Liu et al., 2007) and pulmonary artery SMCs (Xie et al., 2017). The regulation of NFAT or NF- $\chi \mathrm{B}$ on PPAR $\gamma$ promoter activity hasn't been reported in aortic SMCs. Here we showed that both the activity of PPAR $\gamma 2$ promoter and the protein expression of PPAR $\gamma$ decrease in SKI SMCs, which are reversed by either calcineurin inhibitor or NF- $x \mathrm{~B}$ inhibitor, suggesting that PPAR $\gamma 2$ may be a downstream target of calcineurin/NFAT/NF- $\chi \mathrm{B}$ signaling pathways and be negatively regulated, in contrast to the positive regulation of PPAR $\gamma 2$ promoter activity by NFAT in human adipocytes (Kim et al., 2010) and hepatoma cells (Yang et al., 2003). This diversity may be due to different species, cell types, cofactors and other factors, such as various subtypes and concentrations of NFAT.

Though PPAR $\gamma$ could be a downstream target of calcineurin/NFAT/NF- $\varkappa$ B signaling pathways, in return it could also interfere with NFAT and NF- $x$ B (Blanquart et al., 2003). The activated PPAR $\gamma$ physically associates with NFAT and blocks NFAT DNA binding and transcriptional activity in T lymphocyte (Yang et al., 2000). In SMCs, PPAR $\gamma$ inhibits NF- $x \mathrm{~B}$ activity by promoting nuclear export of p65NF- $x$ B, which is abolished by dominant-negative mutation in PPAR $\gamma 1$ (Mukohda et al., 2017). In SKI SMCs, overexpression of PPAR $\gamma 2$ or activation of PPAR $\gamma$ inhibits the expression of phosphorylated p65NF- $\chi \mathrm{B}$, further confirmed the mutual negative regulation between PPAR $\gamma$ and NF- $x$ B. Activation of PPAR $\gamma$ could inhibit the nuclear translocation of NFAT4 and NF- $\varkappa$ B in SKI SMCs. Although NFAT/NF- $x$ B and PPAR $\gamma$ can negatively regulate each other, we infer that inactivation of $\mathrm{C} 674$ firstly activates intracellular $\mathrm{Ca}^{2+}$-dependent calcineurin/NFAT/NF- $\varkappa$ B pathways to suppress PPAR $\gamma$ due to its fundamental function in controlling the concentration of intracellular $\mathrm{Ca}^{2+}$. In return, the down-regulated PPAR $\gamma$ further enhances the nuclear retention of NFAT/NF- $x \mathrm{~B}$, thus shifting the balance between NFAT/NF- $x \mathrm{~B}$ and PPAR $\gamma$ to the continuous activation of NFAT/NF- $x$ B, consequently promoting SMC phenotypic modulation and aortic aneurysm formation. Overexpression or activation of PPAR $\gamma$ could break this vicious circle and thus protect from SMC phenotypic modulation and aortic aneurysm.

Angiotensin II is a common reagent for inducing aortic aneurysms in rodents through complex mechanisms, including activation of calcineurin and NFAT4 in arterial smooth muscle (Nieves-Cintron et al., 2007), activation of NF- $x$ B (Tsai et al., 2017), and down-regulation of PPAR $\curlyvee$ (Subramanian et al., 2012). Activation of PPAR $\gamma$ improves angiotensin II-induced aortic aneurysm (Golledge et al., 2010; Motoki et al., 2015). We have reported that angiotensin II could induce ROS production and cause the irreversible oxidation of C674 in aorta (Que et al., 2020). As data not shown, we found that angiotensin II downregulated PPAR $\gamma$ in SMCs. Our study provided another mechanism for angiotensin II to downregulate PPAR $\gamma$, that is, to activate calcineurin/NFAT/NF- $\varkappa \mathrm{B}$ pathways by causing irreversible oxidization of C674, thereby inhibiting PPAR $r$. Inactivation of C674 in SKI mice might have a synergetic effect with angiotensin II to aggravate aortic aneurysm.

Here, we used a relatively low dose of pioglitazone $\left(15 \mathrm{mg} \cdot \mathrm{kg}^{-1 *}\right.$ day $\left.^{-1}\right)$ instead of the most commonly used dose $\left(50 \mathrm{mg}^{*} \mathrm{~kg}^{-1 *} \mathrm{day}^{-1}\right)$ to activate PPAR rin vivo to minimize its effect on metabolism. Pioglitazone is usually given before the infusion of angiotensin II to prevent the formation of aortic aneurysm (Golledge et al., 2010). In this study, pioglitazone was used 7 days after angiotensin II infusion to observe the preventive and therapeutic effects of PPAR $\curlyvee$ activation on aortic aneurysm. Our results indicate that early treatment with pioglitazone can completely reverse the deterioration of aortic aneurysm in SKI mice, similar to that in WT mice, suggesting that PPAR $\gamma$ activator or SERCA2 activator might be used in the treatment of early aortic aneurysm. CDN1163 is a non-specific SERCA activator (Cornea et al., 2013), and its effect on aortic aneurysm hasn't been reported. However, as the structure of CDN1163 shows the potential of cytotoxicity, there is also a need to develop other chemicals to selectively stimulate SERCA2. At present, we are testing whether CDN1163 and other potential SERCA activators can reverse the phenotypic modulation of SKI SMCs for further application in vivo .

In summary, our data provide direct evidence of the redox status of C674 in SERCA2 in maintaining aortic homeostasis by keeping the balance between NFAT/NF- $\chi \mathrm{B}$ and PPAR $\gamma$. The irreversible oxidative 
inactivation of SERCA2 C674 by increased levels of ROS under aortic aneurysm-prone conditions, such as hypertension and aging, activates $\mathrm{Ca}^{2+}$-dependent calcineurin-mediated NFAT/NF- $x \mathrm{~B}$ pathways that suppresses the expression of PPAR $\gamma$, which in return causes the sustained activation of NFAT/NF- $\varkappa$ B, leads to the phenotypic modulation of SMCs, results in exacerbated aortic aneurysm. Pharmacological activation of PPAR $\gamma$ could restore the balance between NFAT/NF- $x \mathrm{~B}$ and PPAR $\gamma$ to break this vicious circle, thus protecting against SMC phenotypic modulation and aortic aneurysm caused by the inactivation of SERCA2 C674. PPARY and SERCA2 may be potential therapeutic targets for aortic aneurysm.

\section{Acknowledgements}

The plasmids of pLG4.19, pRL-TK renilla, PPAR 2 and CMV-GFP were kindly provided by Dr. Zhongzhou Yang and Dr. Xiaoqiu Xiao. This work was supported by National Natural Science Foundation of China (31571172, 81870343, X.T., and 81700237, P.H.), Chongqing Research Program of Basic Research and Frontier Technology (cstc2016jcyjA0407, X.T.), Fundamental Research Funds for the Central Universities (2018CDQYYX0042, X.T. and 2018CDYXYX0027, P.H.).

\section{Author contributions}

Q., X.S., P. H. and X.T. participated in the research design and performed the data analysis and interpretation; Y.Q. and X.T. wrote the manuscript; Y.Q., X.S., L.W., and S.W. conducted the experiments; All authors reviewed and revised the final version of this manuscript and approved its submission.

\section{Conflict of interest}

The authors declare no conflicts of interest.

\section{Declaration of transparency and scientific rigor}

This Declaration acknowledges that this paper adheres to the principles for transparent reporting and scientific rigor of preclinical research as stated in the BJP guidelines for Design \& Analysis, Immunoblotting, Immunochemistry, and Animal Experimentation, and as recommended by funding agencies, publishers and other organizations engaged with supporting research.

\section{Figure legends}

FIGURE 1 Inactivation of SERCA2 C674 suppresses PPAR $\gamma$ by activation of calcineurin/NFAT/NF- $\varkappa$ B pathways. (a) The main signaling pathways from the Kyoto encyclopedia of genes and genomes pathway enrichment analysis in duplicate aorta samples of WT and SKI mice in LDLR/-background. (b) The mRNA levels of PPAR $\gamma 2$ in aortas of WT and SKI mice in LDLR ${ }^{-/-}$background. (c) Representative Western blots of PPAR $\gamma$ from the aorta of WT and SKI mice in LDLR $/$ - background, and quantification of band intensities in graph. (d) The mouse PPAR $\gamma 2$ promoter activity in aortic SMCs. (e) Effects of CsA or PDTC on PPAR $\gamma 2$ promoter activity in SKI aortic SMCs. (f) Representative Western blots of PPAR $\gamma$ from aortic SMCs and the quantification of band intensities in graph. (b)-(f), mean \pm SEM, unpaired Student's $t$ test, ${ }^{*} P<.05$, significantly different as indicated, $n=5$

FIGURE 2 Activation of NF- $x$ B accounts for the phenotypic modulation of SKI aortic SMCs. (a) Representative Western blots from SKI aortic SMCs treated with PDTC and quantification of band intensities in graph. Mean \pm SEM, unpaired Student's $t$ test, ${ }^{*} P<.05$, significantly different as indicated, $n=5$. (b) Cell proliferation. (c) Cell migration. (d) Macrophage adhesion to aortic SMCs. (b)-(d), mean \pm SEM, unpaired Student's $t$ test, ${ }^{*} P<.05$, significantly different as indicated, $n=6$

FIGURE 3 The down-regulated PPAR 2 accounts for the phenotypic modulation of SKI SMCs. (a) Representative Western blots from SKI aortic SMCs transfected with PPAR ${ }^{2}$ plasmids and quantification of band intensities in graph. Mean \pm SEM, unpaired Student's $t$ test, ${ }^{*} P<.05$, significantly different as indicated, $n=5$. (b) Cell proliferation. (c) Cell migration. (d) Macrophage adhesion to aortic SMCs. (b)-(d), mean \pm SEM, unpaired Student'st test, ${ }^{*} P<.05$, significantly different as indicated, $n=6$ 
FIGURE 4 Activation of PPAR $\gamma$ by pioglitazone prevents the phenotypic modulation of SKI SMCs. (a) Representative Western blots from SKI aortic SMCs treated with pioglitazone and quantification of band intensities in graph. Ctrl, solvent control; PIOG, pioglitazone. Mean \pm SEM, unpaired Student's $t$ test, ${ }^{*} P$ $<.05$, significantly different as indicated, $n=5$. (b) Cell proliferation. (c) Cell migration. (d) Macrophage adhesion to aortic SMCs. (b)-(d), mean \pm SEM, unpaired Student's $t$ test, ${ }^{*} P<.05$, significantly different as indicated, $n=6$

FIGURE 5 Activation of PPAR $\gamma$ represses the activation of NFAT/NF- $\varkappa$ B in SKI aortic SMCs. Representative Western blots of NFAT4 and p65NF- $x$ B from SKI aortic SMCs treated with pioglitazone and quantification of band intensities in graph. Histone $\mathrm{H} 3$ is the load control of nuclear protein and GAPDH is the load control of total protein. Ctrl, solvent control; PIOG, pioglitazone. Mean \pm SEM, unpaired Student's $t$ test, ${ }^{*} P<.05$, significantly different as indicated, $n=5$

FIGURE 6 Activation of PPAR $\curlyvee$ ameliorates angiotensin II-induced aortic aneurysm in SKI mice. (a) The flow chart of animal procedure in $\mathrm{LDLR}^{-/}$background mice. (b) Survival rate. SKI mice treated with solvent control (SKI/Ctrl) vs. WT mice treated with solvent control (WT/Ctrl) or SKI mice treated with pioglitazone (SKI/PIOG), ${ }^{*} P<.05$, Kaplan-Meier analysis and comparisons are performed using the log-rank test. (c) Incidence. (d) Representative photographs of aortic aneurysms and the summary of their composition in graph. A red arrow indicates the location of the aneurysm. Ruptured aneurysm, SKI/Ctrl vs. WT/Ctrl or SKI/PIOG, ${ }^{*} P<.05$, Chi-square test. (b)-(d), $n=11$. (e) Representative photographs of elastin degradation and the percentage of different grades of elastin degradation summarized in graph. Grade IV, SKI/Ctrl vs. WT/Ctrl or SKI/PIOG, ${ }^{*} P<.05$, Chi-square test. (f) Representative photographs of collagen deposition and its score summary in graph. (e)-(f), scale $=500 \mu \mathrm{m}$. Mean \pm SEM, WT/Ctrl, $n$ $=8 ; \mathrm{SKI} / \mathrm{Ctrl}, n=7 ; \mathrm{SKI} / \mathrm{PIOG}, n=9$

FIGURE 7 Activation of PPAR $\gamma$ decreased the size and number of angiotensin II-induced aortic aneurysm in SKI mice. (a) Representative photographs of HE staining of aortic aneurysms and the diameter and area of aneurysm summarized in graph. Scale $=500 \mu \mathrm{m}$. (b) The length of aneurysm. (c) The average number of aortic aneurysms in each group. Mean $\pm \mathrm{SEM}$, One-way ANOVA, ${ }^{*} P<.05$, significantly different as indicated, WT/Ctrl, $n=8 ; \mathrm{SKI} / \mathrm{Ctrl}, n=7 ; \mathrm{SKI} / \mathrm{PIOG}, n=9$

FIGURE 8 Pioglitazone therapy inhibited the expression of aneurysm related proteins in angiotensin IIinfused SKI aorta. (a) Representative Western blots from the aorta of SKI mice in LDLR - $^{-}$background infused with angiotensin II, and quantification of band intensities in graph. Ang II, angiotensin II; Ctrl, solvent control; PIOG, pioglitazone. Mean +- SEM, unpaired Student's $t$ test, ${ }^{*} P<.05$, significantly different as indicated, $n=5$

\section{REFERENCES}

Abe M, Hasegawa K, Wada H, Morimoto T, Yanazume T, Kawamura T, . . . Kita T (2003). GATA-6 is involved in PPARgamma-mediated activation of differentiated phenotype in human vascular smooth muscle cells. Arterioscler Thromb Vasc Biol 23: 404-410.

Adachi T, Weisbrod RM, Pimentel DR, Ying J, Sharov VS, Schoneich C, \& Cohen RA (2004). SGlutathiolation by peroxynitrite activates SERCA during arterial relaxation by nitric oxide. Nat Med $10: 1200-1207$.

Ailawadi G, Moehle CW, Pei H, Walton SP, Yang Z, Kron IL, . . . Owens GK (2009). Smooth muscle phenotypic modulation is an early event in aortic aneurysms. J Thorac Cardiovasc Surg 138: 1392-1399.

Alexander SPH, Kelly E, Mathie A, Peters JA, Veale EL, Armstrong JF, . . . Collaborators C (2019). THE CONCISE GUIDE TO PHARMACOLOGY 2019/20: Introduction and Other Protein Targets. Br J Pharmacol 176 Suppl 1: S1-S20.

Bhamidipati CM, Whatling CA, Mehta GS, Meher AK, Hajzus VA, Su G, . . . Ailawadi G (2014). 5Lipoxygenase pathway in experimental abdominal aortic aneurysms. Arterioscler Thromb Vasc Biol 34: 
2669-2678.

Blanquart C, Barbier O, Fruchart JC, Staels B, \& Glineur C (2003). Peroxisome proliferator-activated receptors: regulation of transcriptional activities and roles in inflammation. J Steroid Biochem Mol Biol 85: 267-273.

Chen R, Yan J, Liu P, \& Wang Z (2015). Effects of thiazolidinedione therapy on inflammatory markers of type 2 diabetes: a meta-analysis of randomized controlled trials. PLoS One 10: e0123703.

Cornea RL, Gruber SJ, Lockamy EL, Muretta JM, Jin D, Chen J, . . . Thomas DD (2013). High-throughput FRET assay yields allosteric SERCA activators. Journal of biomolecular screening 18: 97-107.

Curtis MJ, Bond RA, Spina D, Ahluwalia A, Alexander SP, Giembycz MA, . . . McGrath JC (2015). Experimental design and analysis and their reporting: new guidance for publication in BJP. Br J Pharmacol 172: 3461-3471.

de Winther MP, Kanters E, Kraal G, \& Hofker MH (2005). Nuclear factor kappaB signaling in atherogenesis. Arterioscler Thromb Vasc Biol 25: 904-914.

Golledge J, Cullen B, Rush C, Moran CS, Secomb E, Wood F, . . . Norman PE (2010). Peroxisome proliferator-activated receptor ligands reduce aortic dilatation in a mouse model of aortic aneurysm. Atherosclerosis 210: 51-56.

Golledge J, Muller J, Shephard N, Clancy P, Smallwood L, Moran C, . . . Norman PE (2007). Association between osteopontin and human abdominal aortic aneurysm. Arterioscler Thromb Vasc Biol 27: 655-660.

Halabi CM, Beyer AM, de Lange WJ, Keen HL, Baumbach GL, Faraci FM, \& Sigmund CD (2008). Interference with PPAR gamma function in smooth muscle causes vascular dysfunction and hypertension. Cell Metab 7: 215-226.

Hamblin M, Chang L, Fan Y, Zhang J, \& and Chen Y (2009). PPARs and the cardiovascular system. Antioxid Redox Signal 11: 1415-1452.

Harding SD, Sharman JL, Faccenda E, Southan C, Pawson AJ, Ireland S, . . . Nc I (2018). The IUPHAR/BPS Guide to PHARMACOLOGY in 2018: updates and expansion to encompass the new guide to IMMUNOPHARMACOLOGY. Nucleic Acids Res 46: D1091-D1106.

Hasan DM, Starke RM, Gu H, Wilson K, Chu Y, Chalouhi N, . . . Sigmund CD (2015). Smooth Muscle Peroxisome Proliferator-Activated Receptor gamma Plays a Critical Role in Formation and Rupture of Cerebral Aneurysms in Mice In Vivo. Hypertension 66: 211-220.

Hu Q, Zhang XJ, Zhang C, Zhao YX, He H, Liu CX, . . . Zhang Y (2008). Peroxisome proliferator-activated receptor-gamma1 gene therapy attenuates atherosclerosis and stabilizes plaques in apolipoprotein E-deficient mice. Hum Gene Ther 19: 287-299.

Kim HB, Kumar A, Wang L, Liu GH, Keller SR, Lawrence JC, Jr., . . . Harris TE (2010). Lipin 1 represses NFATc4 transcriptional activity in adipocytes to inhibit secretion of inflammatory factors. Mol Cell Biol 30: 3126-3139.

Li Y, Zhu J, \& Ding JQ (2015). Association of the PPARgamma2 Pro12Ala polymorphism with increased risk of cardiovascular diseases. Genet Mol Res 14: 18662-18674.

Limor R, Sharon O, Knoll E, Many A, Weisinger G, \& Stern N (2008). Lipoxygenase-derived metabolites are regulators of peroxisome proliferator-activated receptor gamma-2 expression in human vascular smooth muscle cells. Am J Hypertens 21: 219-223.

Liu G, Wu F, Jiang X, Que Y, Qin Z, Hu P, . . . Tong X (2020). Inactivation of Cys(674) in SERCA2 increases BP by inducing endoplasmic reticulum stress and soluble epoxide hydrolase. Br J Pharmacol 177: 1793-1805. 
Liu L, \& Clipstone NA (2007). Prostaglandin F2alpha inhibits adipocyte differentiation via a G alpha q-calcium-calcineurin-dependent signaling pathway. J Cell Biochem 100: 161-173.

Meng YH, Tian C, Liu L, Wang L, \& Chang Q (2014). Elevated expression of connective tissue growth factor, osteopontin and increased collagen content in human ascending thoracic aortic aneurysms. Vascular 22: $20-27$.

Meredith D, Panchatcharam M, Miriyala S, Tsai YS, Morris AJ, Maeda N, . . . Smyth SS (2009). Dominantnegative loss of PPARgamma function enhances smooth muscle cell proliferation, migration, and vascular remodeling. Arterioscler Thromb Vasc Biol 29: 465-471.

Miyake T, Aoki M, Masaki H, Kawasaki T, Oishi M, Kataoka K, . . . Morishita R (2007). Regression of abdominal aortic aneurysms by simultaneous inhibition of nuclear factor kappaB and ets in a rabbit model. Circ Res 101: 1175-1184.

Monaco C, \& Paleolog E (2004). Nuclear factor kappaB: a potential therapeutic target in atherosclerosis and thrombosis. Cardiovasc Res 61: 671-682.

Motoki T, Kurobe H, Hirata Y, Nakayama T, Kinoshita H, Rocco KA, . . . Kitagawa T (2015). PPARgamma agonist attenuates inflammation in aortic aneurysm patients. Gen Thorac Cardiovasc Surg 63: 565-571.

Mukohda M, Lu KT, Guo DF, Wu J, Keen HL, Liu X, . . . Sigmund CD (2017). Hypertension-Causing Mutation in Peroxisome Proliferator-Activated Receptor gamma Impairs Nuclear Export of Nuclear FactorkappaB p65 in Vascular Smooth Muscle. Hypertension 70: 174-182.

Nakao T, Horie T, Baba O, Nishiga M, Nishino T, Izuhara M, . . . Ono K (2017). Genetic Ablation of MicroRNA-33 Attenuates Inflammation and Abdominal Aortic Aneurysm Formation via Several AntiInflammatory Pathways. Arterioscler Thromb Vasc Biol 37: 2161-2170.

Nieves-Cintron M, Amberg GC, Nichols CB, Molkentin JD, \& Santana LF (2007). Activation of NFATc3 down-regulates the beta1 subunit of large conductance, calcium-activated $\mathrm{K}+$ channels in arterial smooth muscle and contributes to hypertension. J Biol Chem 282: 3231-3240.

Qin F, Siwik DA, Lancel S, Zhang J, Kuster GM, Luptak I, . . . Colucci WS (2013). Hydrogen peroxidemediated SERCA cysteine 674 oxidation contributes to impaired cardiac myocyte relaxation in senescent mouse heart. J Am Heart Assoc 2: e000184.

Que Y, Shu X, Wang L, Hu P, Wang S, Xiong R, . . . Tong X (2020). Inactivation of cysteine 674 in the SERCA2 accelerates experimental aortic aneurysm. J Mol Cell Cardiol.

Serfling E, Berberich-Siebelt F, Avots A, Chuvpilo S, Klein-Hessling S, Jha MK, . . . Palmetshofer A (2004). NFAT and NF-kappaB factors-the distant relatives. Int J Biochem Cell Biol 36: 1166-1170.

Shimada K, Furukawa H, Wada K, Korai M, Wei Y, Tada Y, . . . Hashimoto T (2015). Protective Role of Peroxisome Proliferator-Activated Receptor-gamma in the Development of Intracranial Aneurysm Rupture. Stroke 46: 1664-1672.

Subramanian V, Golledge J, Heywood EB, Bruemmer D, \& Daugherty A (2012). Regulation of peroxisome proliferator-activated receptor-gamma by angiotensin II via transforming growth factor-beta1-activated p38 mitogen-activated protein kinase in aortic smooth muscle cells. Arterioscler Thromb Vasc Biol 32: 397-405.

Tang RH, Zheng XL, Callis TE, Stansfield WE, He J, Baldwin AS, . . . Selzman CH (2008). Myocardin inhibits cellular proliferation by inhibiting NF-kappaB(p65)-dependent cell cycle progression. Proc Natl Acad Sci U S A 105: 3362-3367.

Thompson MD, Mei Y, Weisbrod RM, Silver M, Shukla PC, Bolotina VM, . . . Tong X (2014). Glutathione adducts on sarcoplasmic/endoplasmic reticulum Ca2+ ATPase Cys-674 regulate endothelial cell calcium 
stores and angiogenic function as well as promote ischemic blood flow recovery. J Biol Chem 289: 1990719916.

Tong X, Khandelwal AR, Qin Z, Wu X, Chen L, Ago T, . . . Cohen RA (2015). Role of smooth muscle Nox4-based NADPH oxidase in neointimal hyperplasia. J Mol Cell Cardiol 89: 185-194.

Tong X, Khandelwal AR, Wu X, Xu Z, Yu W, Chen C, . . Zeng C (2016). Pro-atherogenic role of smooth muscle Nox4-based NADPH oxidase. J Mol Cell Cardiol 92: 30-40.

Tong X, Ying J, Pimentel DR, Trucillo M, Adachi T, \& Cohen RA (2008). High glucose oxidizes SERCA cysteine- 674 and prevents inhibition by nitric oxide of smooth muscle cell migration. J Mol Cell Cardiol 44: 361-369.

Tsai MH, Lee CW, Hsu LF, Li SY, Chiang YC, Lee MH, . . . Lee IT (2017). CO-releasing molecules CORM2 attenuates angiotensin II-induced human aortic smooth muscle cell migration through inhibition of ROS/IL-6 generation and matrix metalloproteinases-9 expression. Redox Biol 12: 377-388.

Wang L, Zhang J, Fu W, Guo D, Jiang J, \& Wang Y (2012a). Association of smooth muscle cell phenotypes with extracellular matrix disorders in thoracic aortic dissection. J Vasc Surg 56: 1698-1709, 1709 e1691.

Wang S, Zhang C, Zhang M, Liang B, Zhu H, Lee J, . . . Zou MH (2012b). Activation of AMP-activated protein kinase alpha2 by nicotine instigates formation of abdominal aortic aneurysms in mice in vivo. Nat Med 18: 902-910.

Werman A, Hollenberg A, Solanes G, Bjorbaek C, Vidal-Puig AJ, \& Flier JS (1997). Ligand-independent activation domain in the $\mathrm{N}$ terminus of peroxisome proliferator-activated receptor gamma (PPARgamma). Differential activity of PPARgamma1 and -2 isoforms and influence of insulin. J Biol Chem 272: 20230-20235.

Xie X, Li S, Zhu Y, Liu L, Pan Y, Wang J, . . . Li M (2017). MicroRNA-27a/b mediates endothelin-1induced PPARgamma reduction and proliferation of pulmonary artery smooth muscle cells. Cell Tissue Res 369: 527-539.

Yang TT, \& Chow CW (2003). Transcription cooperation by NFAT.C/EBP composite enhancer complex. J Biol Chem 278: 15874-15885.

Yang XY, Wang LH, Chen T, Hodge DR, Resau JH, DaSilva L, \& Farrar WL (2000). Activation of human T lymphocytes is inhibited by peroxisome proliferator-activated receptor gamma (PPARgamma) agonists. PPARgamma co-association with transcription factor NFAT. J Biol Chem 275:4541-4544.

Ying J, Sharov V, Xu S, Jiang B, Gerrity R, Schoneich C, \& Cohen RA (2008). Cysteine-674 oxidation and degradation of sarcoplasmic reticulum $\mathrm{Ca}(2+)$ ATPase in diabetic pig aorta. Free Radic Biol Med 45:756-762.

\section{Hosted file}

SKI-AA-BJP-Table.docx available at https://authorea.com/users/353973/articles/477687inactivation-of-serca2-cys674-accelerates-aortic-aneurysms-by-suppressing-ppar\%CE\%B3 
(a)

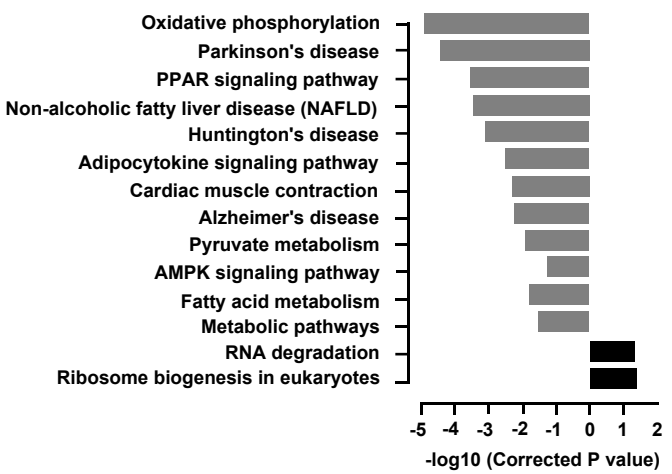

(b)

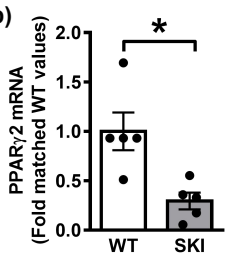

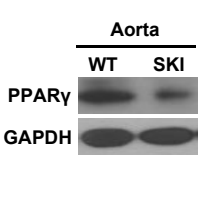

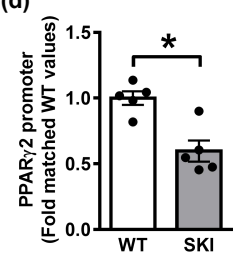

(e)

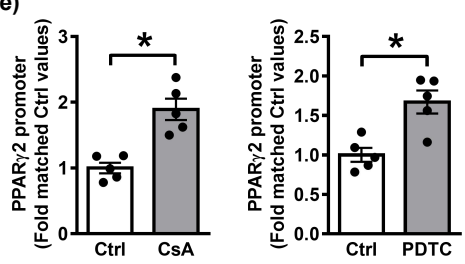

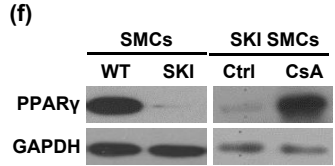

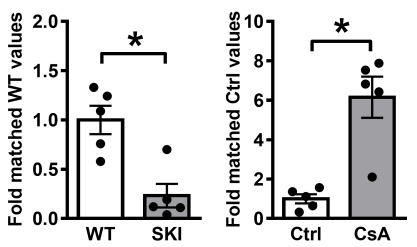

Figure 1 